\section{A patient with polytrauma, hypothermia and cardiac arrest after delayed mountain rescue}

With interest, we followed the case report by Ting and Brown ${ }^{1}$ of a severely hypothermic patient with trauma treated with extracorporeal life support. We fully agree with the authors that extracorporeal life support should be taken into consideration in distinct cases of multiple trauma and severe hypothermia. We report a case of a hypothermic patient with polytrauma who arrived in cardiac arrest after a fall of $30 \mathrm{~m}$ in the mountains. ${ }^{2}$

Owing to bad weather, the helicopter emergency medical service team had reached the 59-year-old man four hours after the fall occurred. The patient had signs of cardiac instability and a Glasgow Coma Scale of 9/15. During transport to the level I trauma centre, he sustained cardiac arrest. On arrival, he was undergoing cardiopulmonary resuscitation, and had ventricular fibrillation, a core temperature of $25.3^{\circ} \mathrm{C}$ and a potassium level of $2.7 \mathrm{mmol} / \mathrm{L}$.

The receiving team decided to proceed according to our Bernese Hypothermia Algorithm ${ }^{3}$ and to rewarm the patient, with limited trauma assessment. The assessment, which was done simultaneously with cannulation of the groin, included a primary survey and extended focused assessment with sonography in trauma to exclude major hemorrhage. As large bleeding was excluded, the team decided to start active invasive rewarming using minimal extracorporeal circulation. ${ }^{4}$ During rewarming, the patient underwent a full trauma assessment using our local polytrauma protocol. The contrast computed tomography scan among others - showed serial rib frac- tures with hemopneumothorax, an open book pelvic fracture, a pertrochanteric femur fracture and an active hemorrhage from a lumbar artery. The arterial bleeding was successfully embolized. Subsequently, the patient underwent several orthopedic interventions. Fourteen days after the fall, he was discharged home with full neurologic recovery.

Even in otherwise usually hopeless combinations of cardiac arrest in multiple trauma, a good outcome is possible if the cardiac arrest is caused by accidental hypothermia, as in our case. We hope to motivate colleagues to apply the concept of "no one is dead until he is warm and dead"5 even in patients with polytrauma, because rewarming could be the only strategy to improve coagulation and allow survival. ${ }^{6}$

\section{Monika Brodmann Maeder MD MME}

Emergency physician, Department of Emergency Medicine, Bern University Hospital and Institute of Mountain Emergency Medicine, EURAC rescue, Bozen, Italy

\section{Volker Lischke MD}

Anesthesiologist, Department of Anaesthesiology and Intensive Care Medicine, Hochtaunus-Kliniken gGmbH, Bad Homburg, Germany; German Red Cross Mountain Rescue, Berlin, Germany

\section{Armin Berner MD}

Anesthesiologist, Department of Anesthesia and Surgical Intensive Care, Hospital Garmisch-Partenkirchen, Garmisch-Partenkirchen, Germany; Mountain Rescue Bavaria, Bad Tolz, Germany

\section{Oliver Reisten MD PhD}

Anesthesiologist, Emergency Medical Service, Zermatt, Switzerland, and International Commission for Mountain Emergency Medicine (ICAR MEDCOM)

\section{Urs Pietsch MD}

Anesthesiologist, Department of Anaesthesiology and Intensive Care Medicine, Cantonal Hospital, St. Gallen, Switzerland; Air Zermatt, Zermatt, Switzerland

\section{Mathieu Pasquier MD}

Emergency physician, Emergency Service, Lausanne University Hospital Center, CHUV, Lausanne, Switzerland; International Commission for Mountain Emergency Medicine

Cite as: CMAJ 2018 October 22;190: E1263. doi: $10.1503 / \mathrm{cmaj} .70338$

\section{References}

1. Ting DK, Brown DJ. Use of extracorporeal life support for active rewarming in a hypothermic, nonarrested patient with multiple trauma. CMAJ 2018;190:E718-21

2. Lischke V, Berner A, Pietsch U, et al. Cardiac arrest in mountain areas during winter season. Der Notarzt 2014;30:58-65.

3. Monika BM, Martin D, Balthasar E, et al. The Bernese Hypothermia Algorithm: a consensus paper on in-hospital decision-making and treatment of patients in hypothermic cardiac arrest at an alpine level 1 trauma centre. Injury 2011;42:539-43.

4. Anastasiadis K, Murkin J, Antonitsis P, et al. Use of minimal invasive extracorporeal circulation in cardiac surgery: principles, definitions and potential benefits. A position paper from the Minimal invasive Extra-Corporeal Technologies international Society (MiECTiS). Interact Cardiovasc Thorac Surg 2016;22:647-62.

5. Hilmo J, Naesheim T, Gilbert M. "Nobody is dead until warm and dead": prolonged resuscitation is warranted in arrested hypothermic victims also in remote areas - a retrospective study from northern Norway. Resuscitation 2014;85:1204-11.

6. Paal P, Gordon L, Strapazzon G, et al. Accidental hypothermia-an update: The content of this review is endorsed by the International Commission for Mountain Emergency Medicine (ICAR MEDCOM). Scand J Trauma Resusc Emerg Med 2016;24:111.

Competing interests: None declared.

The authors have obtained patient consent. 